\title{
Contemporary treatment of metastatic renal cell carcinoma
}

\author{
Igor Stukalin, Nimira Alimohamed, Daniel Y.C. Heng \\ Department of Medical Oncology, Tom Baker Cancer Centre, University of Calgary, Calgary, AB, \\ Canada
}

\begin{abstract}
The introduction of targeted therapy has revolutionized the treatment of patients with metastatic renal cell carcinoma (mRCC). The current standard of care focuses on the inhibition of angiogenesis through the targeting of the vascular endothelial growth factor receptor (VEGFR) and the mammalian target of rapamycin (mTOR). Over the past few years, research exploring novel targeted agents has blossomed, leading to the approval of various targeted therapies. Furthermore, results from the CheckMate025 and the METEOR trials have brought about two additional novel options: the programmed cell death 1 (PD-1) checkpoint inhibitor nivolumab and the MET/NEGFR/AXL inhibitor cabozantinib, respectively. With the variety of therapeutic agents available for treatment of mRCC, research examining appropriate sequencing and combinations of the drugs is ongoing.

This review discusses the role of prognostic criteria, such as those from the International Metastatic Renal Cell Carcinoma Database Consortium (IMDC) criteria. It also covers the current standard of treatment for mRCC with targeted therapy in first-, second-, and thirdline setting. Additionally, the novel mechanism of action of nivolumab and cabozantinib, therapeutic sequencing and ongoing clinical trials are discussed.
\end{abstract}

Correspondence: Daniel Y.C. Heng, Tom Baker Cancer Center, University of Calgary, 1331 29th Street NW, Calgary, AB, T2N 4N2, Canada.

Tel.: +1.403.521.3166 - Fax: +1.403.283.1651.

E-mail: Daniel.heng@albertahealthservices.ca

Key words: Renal cell carcinoma; targeted therapy; immunotherapy; nivolumab; cabozantinib.

Contributions: the authors contributed equally.

Conflict of interest: IS and NA declare no potential conflict of interest; DYCH declares consulting or advisory roles for BMS, Exelixis, Pfizer, Novartis.

Received for publication: 23 March 2016.

Revision received: 30 May 2016.

Accepted for publication: 29 June 2016.

This work is licensed under a Creative Commons Attribution NonCommercial 4.0 License (CC BY-NC 4.0).

(C) Copyright I. Stukalin et al., 2016

Licensee PAGEPress, Italy

Oncology Reviews 2016; 10:295

doi:10.4081/oncol.2016.295

\section{Introduction}

Kidney cancer is not an uncommon malignancy, with an estimated worldwide annual incidence of about 270,000 new cases. ${ }^{1,2}$ Approximately $90 \%$ of kidney cancers are renal cell carcinoma (RCC). ${ }^{2}$ For unknown reasons the overall incidence of metastatic RCC (mRCC) continues to rise by $2 \%$ per year. ${ }^{3} 25-30 \%$ of patients initially present with metastatic disease, while approximately $30 \%$ of patients who are treated for local RCC will relapse. ${ }^{4,5}$ Therapeutic intervention has moved away from cytokine immunotherapeutic agents, such as interferon- $\alpha$ (IFN- $\alpha$ ) and interleukin-2, towards addressing molecular targets through targeted agents. Over the past decade, therapy for mRCC has been revolutionized through the introduction of mammalian target of rapamycin (mTOR) inhibitors and tyrosine kinase inhibitors (TKI), which target the vascular endothelial growth factor (VEGF) pathway. ${ }^{6,7}$ Before this year, there were seven targeted agents approved for therapeutic use in mRCC patients: sunitinib, sorafenib, pazopanib, bevacizumab and axitinib, which target the VEGF pathway and the two mTOR inhibitors everolimus and temsirolimus. ${ }^{3}$ Furthermore, recent clinical trials have identified cabozantinib, an inhibitor of VEGFR receptor (VEGFR), MET and AXL, and nivolumab, a programmed cell death-1 (PD1) checkpoint inhibitor, as novel targeted agents against mRCC. 8,9

\section{Importance of prognostic factors}

With the rapid developments in the mRCC field it remains critical to use prognostic factors, such as the International Metastatic Renal Cell Carcinoma Database Consortium (IMDC) prognostic criteria, to evaluate and identify the best possible treatment methods for different patient subgroups. ${ }^{10,11}$

A prognostic factor is a clinical or biologic characteristic, which allows for an objective determination of a possible outcome of the disease. ${ }^{11}$ Currently, prognostic models addressing mRCC, such as the IMDC prognostic factors and the Memorial Sloan-Kettering Cancer Centre (MSKCC) prognostic criteria, are commonly used in the clinical and research setting. ${ }^{10,12}$ The MSKCC model was validated during the cytokine therapy era, whereas the IMDC model has been validated and used in the setting of targeted therapy. Moreover, the same IMDC model has been validated in first- and second-line targeted therapy, while also displaying the ability to risk-stratify patients in third-line targeted therapy setting. ${ }^{10,13,14}$

The IMDC model includes the following factors: Karnofsky performance status (KPS) $<80 \%$, time from diagnosis to treatment $<1$ year, anemia, hypercalcemia, neutrophilia and thrombocytosis. ${ }^{10}$ Patients with 0 risk factors, 1-2 factors, or $>3$ factors are considered favorable, intermediate, or poor risk, with a corresponding predicted overall survival (OS) of 43.2 months 22.5 months and 7.8 months, respectively. ${ }^{10,15}$

The IMDC prognostic factors have been increasingly used to stratify 
patients into different risk groups in a variety of clinical trials, such as the ADAPT trial (NCT01582672), which examined the effectiveness of autologous dendritic cell immunotherapy (AGS-003) in addition to sunitinib in mRCC. ${ }^{16}$ The IMDC model was also used to stratify patients in the CheckMate214 trial (NCT 02231749) comparing sunitinib versus nivolumab in combination with ipilimumab in first-line setting. ${ }^{17}$

Prognostic factors carry importance in patient counseling and allow clinicians to have a survival reference point based on the risk profiles of these patients. It is important to note that prognosis is a dynamic process, meaning that prognostic prospects of patients can change over the course of the disease. ${ }^{11}$ For example, with increasing information gathered on a patient who has survived a certain period of time, the prognosis of the patient may be different than what it originally was at baseline.

Prognostic factors can also aid in treatment selection. Patients with a poor risk factor profile would be eligible for the first-line mTOR inhibitor temsirolimus. ${ }^{18}$ Temsirolimus was tested in 626 patients with previously untreated, poor-prognosis mRCC. ${ }^{18}$ Patients were randomized to temsirolimus $25 \mathrm{mg}$ IV weekly, IFN- $\alpha 18 \times 10^{6}$ IU times weekly, or temsirolimus + IFN- $\alpha 3$ times weekly. ${ }^{18}$ Results showed that patients receiving temsirolimus alone had a longer $\mathrm{OS}$ of 10.9 months relative to 7.3 months $(\mathrm{P}=0.008)$ in the IFN- $\alpha$ group. ${ }^{18}$ As a result, the use of prognostic factors remains critical to identify the subgroup of poor risk mRCC patients.

Small subgroups of favorable risk mRCC patients may follow an indolent clinical course characterized by very slow tumor growth and no significant symptoms. ${ }^{11}$ This small patient subgroup may benefit from active surveillance with regularly scheduled computed tomography scans to monitor their tumor growth. ${ }^{19}$ Although a variety of clinical trials have displayed a significant survival benefit associated with the current targeted agents, they induce significant toxicities. A retrospective cohort study of patients treated at two centers for mRCC evaluated clinical outcomes in patients treated with deliberately deferred firstline targeted therapy. ${ }^{19}$ In this study patients who were observed for an average of 18.7 months prior to treatment initiation had progression free survival (PFS) and OS comparable to those observed in the phase III clinical trial testing sunitinib versus IFN- $\alpha .{ }^{20}$ Moreover, data from a handful of retrospective and prospective studies confirmed these results by displaying that subgroups of favorable and intermediate risk patients may benefit from active surveillance. ${ }^{21-23}$ Therefore, patients with slow tumor growth may be able to avoid being exposed to the toxicities associated with the targeted therapies, without lowering their survival benefit by going on active surveillance.

The IMDC model can also be used to help select patients that would benefit the most from a cytoreductive nephrectomy (CN); the resection of the primary tumor in mRCC patients. ${ }^{24}$ Patients with four or more IMDC prognostic factors appear to be less likely to benefit from $\mathrm{CN}$, because the incremental benefit of $\mathrm{CN}$ appears to decrease in patients with a shorter predicted survival. ${ }^{24}$ Risk stratification for $\mathrm{CN}$ is aided with the use of the IMDC model however, it should not be used in isolation for clinical decision making since additional factors, such as brain metastasis, liver metastasis, the bulk of disease in the kidney versus systemically, and surgical resectability may influence whether or not patients would benefit from $\mathrm{CN} .^{24}$

\section{First-line therapy}

\section{Sunitinib}

After displaying superiority to cytokine immunotherapy, the multikinase inhibitor sunitinib that blocks VEGFRs 1,2 and 3, platelet-derived growth factor (PDGF) receptor- $\beta$ and related receptor tyrosine kinases (RTKs) has remained a standard of care for first-line therapy in
mRCC..$^{20,25}$ Results from a phase III randomized controlled trial displayed an objective response rate (ORR) of $31 \%$ versus $6 \%(\mathrm{P}<0.001) .{ }^{20}$ The PFS was 11 months in the sunitinib group versus 5 months in the IFN- $\alpha$, corresponding to a hazard ratio (HR) of 0.42 [95\% confidence interval (CI) $0.32-0.54 ; \mathrm{P}<0.001]$, thus displaying a significant survival benefit associated with first-line suntinib. ${ }^{20}$ The dose and schedule optimization of sunitinib continues to be under evaluation as seen in a prospective phase II study examining toxicity-driven dosages of sunitinib (NCT01499121), which aimed to expose patients to $\leq$ grade-2 toxicity. ${ }^{26}$ Patients were started on $50 \mathrm{mg} /$ day (d) on a $28 / 7 \mathrm{~d}$ schedule. ${ }^{26}$ If the patient had $>$ grade 2 toxicities, the schedule of sunitinib was altered first instead of altering the dose (e.g., 2 weeks on 1 week off) in an effort to maximize dose intensity while maintaining a manageable and tolerable toxicity profile. ${ }^{26}$ Patients who had no toxicities were dose escalated to $62.5 \mathrm{mg}$ and then $75 \mathrm{mg}$ on a $14 / 7 \mathrm{~d}$ schedule. ${ }^{26}$ Patients treated in this manner had an impressive ORR of 50.6\% and a disease control rate of $89.2 \%{ }^{26}$ The benefit of the latter approach remains to be studied further.

\section{Pazopanib}

Pazopanib is a TKI of VEGFRs 1-3, PDGF receptors $\alpha$ and $\beta$, and the proto-oncogene c-Kit. ${ }^{27}$ Pazopanib has previously been shown to significantly prolong PFS and ORR when compared with placebo in both, treatment-naïve and cytokine-pretreated patients with advanced RCC. ${ }^{27} \mathrm{~A} \mathrm{PFS}$ of 9.2 versus 4.2 months $(\mathrm{P}<0.0001)$ and an $0 \mathrm{RR}$ of $30 \%$ versus $3 \%$ $(\mathrm{P}<0.001)$ was observed in the pazopanib and placebo groups, respectively. ${ }^{27}$ Due to these promising results in first-line setting, the COMPARZ trial examined pazopanib versus the first-line standard of care TKI, sunitinib. ${ }^{28}$ Of patients 1110 with clear-cell $\mathrm{mRCC}$ were randomized to receive pazopanib $800 \mathrm{mg}$ once daily or sunitinib in 6-week cycles (50 mg once daily for 4 weeks, followed by 2 weeks off treatment). ${ }^{28}$ Results confirmed pazopanib's noninferiority to sunitinib in terms of PFS. ${ }^{28}$ Furthermore, pazopanib appears to have a favorable safety and qualityof-life profile in comparison to sunitinib. ${ }^{28}$ ORR and OS did not differ significantly between the two treatment groups. ${ }^{28}$ Adverse events, such as fatigue, hand-foot syndrome and mouth sores occurred more frequently in the sunitinib group. ${ }^{28}$ In fact, patients who received pazopanib reported less fatigue, less soreness of extremities and of the mouth and throat but there was a higher incidence of liver toxicity. ${ }^{28}$ Additionally, the PISCES trial evaluated patient preference for pazopanib or sunitinib. Patients were randomly assigned to receive pazopanib $800 \mathrm{mg} / \mathrm{d}$ for 100 weeks, a 2-week washout, then sunitinib $50 \mathrm{mg} / \mathrm{d}$ ( 4 weeks on/2 off/4 on), or the reverse sequence. ${ }^{29} 70 \%$ of patients preferred pazopanib over sunitinib, $22 \%$ preferred sunitinib, while $8 \%$ had no preference $(\mathrm{P}<0.001)$, thereby further confirming patient preference for pazopanib. ${ }^{29}$ Thus, pazopanib is another first-line treatment option.

\section{Bevacizumab}

Bevacizumab is a recombinant monoclonal antibody, which targets and binds circulating VEGF. ${ }^{30}$ Bevacizumab + IFN- $\alpha$ had a significantly higher PFS than IFN- $\alpha$ alone of 10.2 months versus 5.4 months $(\mathrm{P}<0.0001)$ and an $0 R R$ of 31 versus $13 \%(\mathrm{P}<0.0001)$, respectively. ${ }^{30}$ There was no significant OS difference seen between the two groups. Currently there is no data available to determine whether bevacizumab alone or bevacizumab + IFN- $\alpha$ has a larger survival benefit. ${ }^{30}$

\section{Second-line therapy}

\section{Axitinib}

Axitinib is a selective second-generation inhibitor of VEGFR 1, 2 and 3 , while also inhibiting PDGF- $\alpha$, PDGF- $\beta$ and the proto oncogene C- 
Kit. ${ }^{31-33}$ Patients were randomly assigned to receive either axitinib or sorafenib after previous treatment with sunitinib, bevacizumab, temsirolimus, or cytokines. ${ }^{31}$ The primary endpoint was PFS, which was 6.7 months and 4.7 months $(\mathrm{P}<0.0001)$ for axitinib and sorafenib, respectively. ${ }^{31}$ Common toxicities associated with axitinib were diarrhea, hypertension and fatigue. ${ }^{31}$ ORR was $19 \%$ for axitinib and $9 \%$ for sorafenib $(\mathrm{P}=0.0001) .{ }^{31} \mathrm{OS}$ was not significantly different between the two drugs. ${ }^{34}$ As a result axitinib is a second-line treatment option in mRCC patients (Table 1).

\section{Cabozantinib}

Cabozantinib inhibits the activity of VEGFR 1,2,3, hepatocyte growth factor receptor (MET), AXL, the angiopoetin receptor TIE-2, RET, c-Kit and FLT- 3 in vitro and in vivo., $85-38$ These receptors are mainly involved in tumor oncogenesis and angiogenesis. ${ }^{36,38}$ Chronic sunitinib treatment of RCC in vitro has displayed AXL and MET up-regulation, leading to increased prometastatic behavior and angiogenesis. ${ }^{39}$ As a result, cabozantinib's novel mechanism of action could be used to override this resistance mechanism (Figure 1). In preclinical models with neuroendocrine, breast, pancreatic, lung, and glioma tumors, cabozantinib has also been shown to inhibit angiogenesis, tumor cell migration, tumor cell proliferation, while inducing cell death. ${ }^{38,40}$

Hypoxic tumor cells activate hypoxia inducible factor- $1 \alpha$, which leads to the upregulation of both VEGF and MET. ${ }^{41,42}$ The upregulation of MET allows for more hepatocyte growth factor (HGF) to bind to the MET receptor, thereby increasing the invasiveness and motility of the tumor. ${ }^{42,43}$ The increased expression of VEGF increases angiogenesis around the hypoxic tumor cells. ${ }^{42,44}$ Continuous VEGF pathway inhibition through TKIs has been shown to initially slow tumor growth by decreasing the vasculature surrounding the tumor. ${ }^{7,42}$ However, after a period of time the majority of patients develop resistance to VEGF-inhibtion. ${ }^{45,46}$ In fact, the HGF/MET pathway has been identified to act as antiangiogenic escape mechanism in sunitinib-resistant tumors. ${ }^{47}$ Furthermore, MET activation has previously been shown to be correlated with worse disease-specific survival. ${ }^{48}$ As a result, cabozantinib's novel mechanism of action targeting VEGFR, MET and AXL may be advantageous in patients who have developed resistance to previous TKIs.

Table 1. Selected clinical trials of second-line targeted therapies in renal cell carcinoma.

\begin{tabular}{|c|c|c|c|c|c|}
\hline Agent & Mechanism & Trial arms & ORR (\%) & PFS (Months) & OS (Months) \\
\hline Axitinib & VEGFR inhibitor & $\begin{array}{l}\text { Axitinib versus } \\
\text { sorafenib }\end{array}$ & $\begin{array}{c}19 \text { versus } 9 \\
P=0.0001\end{array}$ & $\begin{array}{c}6.7 \text { versus } 4.7 \\
P<0.0001\end{array}$ & $\begin{array}{c}20.1 \text { versus } 19.2 \\
\mathrm{P}=0.3744 \\
\mathrm{NS}\end{array}$ \\
\hline Cabozantinib & $\begin{array}{c}\text { VEGF, MET } \\
\text { and AXL inhibitor }\end{array}$ & $\begin{array}{c}\text { Cabozantinib versus } \\
\text { everolimus }\end{array}$ & $\begin{array}{l}21 \text { versus } 5 \\
P<0.001\end{array}$ & $\begin{array}{l}7.5 \text { versus } 3.9 \\
\mathrm{P}<0.001\end{array}$ & $\begin{array}{c}21.4 \text { versus } 16.5 \\
P<0.001\end{array}$ \\
\hline Nivolumab & PD-1 inhibitor & $\begin{array}{l}\text { Nivolumab versus } \\
\text { everolimus }\end{array}$ & $\begin{array}{c}25 \text { versus } 5 \\
\mathrm{P}<0.001\end{array}$ & $\begin{array}{c}4.6 \text { versus } 4.4 \\
\mathrm{P}=0.11 \\
\mathrm{NS}\end{array}$ & $\begin{array}{c}25.0 \text { versus } 19.6 \\
P=0.002\end{array}$ \\
\hline
\end{tabular}

ORR, objective response rates; PFS, progression free survival; OS, overall survival; VEGFR, vascular endothelial growth factor receptor; NS, non-significant; PD-1, programmed cell death-1.

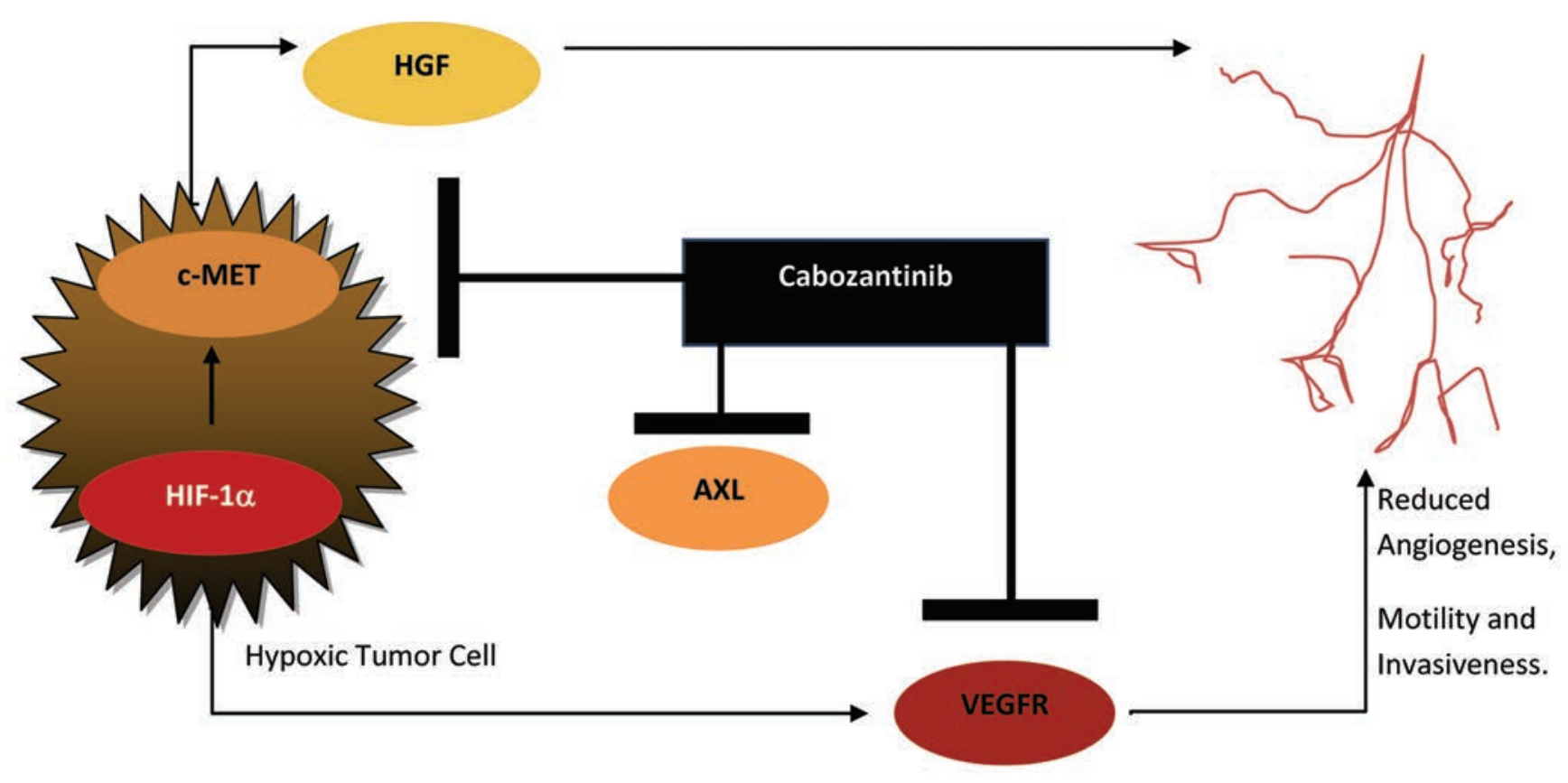

Figure 1. Mechanism of action of cabozantinib: cabozantinib inhibits the activity of c-MET, vascular endothelial growth factor receptor (VEGFR), AXL and other tyrosine kinases, thereby leading to reduced tumor angiogenesis, motility and invasiveness. HGF, hepatocyte growth factor; HIF-1 $\alpha$, hypoxia-inducible factor $1-\alpha$. 
Cabozantinib has already gained the U.S. Food and Drug Administration (FDA) approval for treatment of medullary thyroid cancer. ${ }^{49}$ In mRCC, the METEOR trial randomly assigned 658 patients who had previously received at least one VEGFR-TKI to receive either cabozantinib at $60 \mathrm{mg}$ daily or everolimus at $10 \mathrm{mg}$ daily. ${ }^{8}$ Patients were required to have a KPS of at least $70 \%$ and previous mTOR inhibition therapy was not permitted. ${ }^{8}$ Dose reductions for cabozantinib (40 mg, then $20 \mathrm{mg}$ ) and everolimus (5 $\mathrm{mg}$ then $2.5 \mathrm{mg}$ ) and interruptions of study treatment were allowed to manage adverse events. ${ }^{8}$ The primary end-point was PFS, which was 7.4 months and 3.8 months with cabozantinib and everolimus, respectively. ${ }^{8}$ The 0 RR was $21 \%$ with cabozantinib and $5 \%$ with everolimus $(\mathrm{P}<0.001) .{ }^{8} \mathrm{OS}$ was initially reported in an interim analysis, which displayed longer survival in the cabozantinib than in the everolimus group (HR: 0.67; $\mathrm{P}=0.005){ }^{8}$ The median OS was 21.4 months for the cabozantinib group and 16.5 months for the everolimus group, with a $33 \%$ reduction in the rate of death (HR $0.67,95 \%$ CI 0.53 $0.83, \mathrm{P}=0.0003) .{ }^{50}$ Cabozantinib appears to be the only currently available targeted therapy that significantly increases ORR, PFS and OS in mRCC patients. ${ }^{8,50}$ Frequent toxicities associated with cabozantinib were diarrhea, palmar-plantar erythrodysesthesia syndrome and dysgeusia. ${ }^{8}$ Of patients $60 \%$ who received cabozantinib and $25 \%$ who received everolimus underwent dose reductions. ${ }^{8}$ The incidence of grade 3 and 4 all-cause adverse events was higher in the cabozantinib group (68\%) versus the everolimus group (58\%). ${ }^{8}$ Although a significant survival benefit was observed, cabozantinib dosing needs to be titrated carefully to reduce the number of adverse events seen in the METEOR trial. ${ }^{8}$

\section{Nivolumab}

Nivolumab is a fully human IgG4 PD-1 immune checkpoint inhibitor antibody that inhibits the interaction between PD-1 expressed on acti- vated T-cells and PD-1 ligand (PD-L1) and PD-L2, which are expressed tumor cells. ${ }^{51,52}$ Other PD-1 and PD-L1 inhibitors are also under development, including the PD-1 inhibitor pembrolizumab and the PD-L1 inhibitors atezolizumab and avelumab. T-cells normally recognize tumors through the tumor specific antigens docking to the T-cell receptors (Figure 2). T-cell activation requires both antigen recognition and antigen-independent co-regulatory signaling to occur. ${ }^{53}$ Immune checkpoint pathways, such as PD-1 oversee the co-regulatory signaling step. ${ }^{54}$ The cell surface receptor PD-1 belongs to the CD28 family of Tcell regulators, which are expressed on activated T-cells and other immune cells. ${ }^{54,55}$ When PD-1 interacts with its ligands, PD-L1 and PDL2, T-cells are switched off resulting in T-cell exhaustion, thereby significantly down-regulating immune response even in the presence of antigens that would normally trigger an immune response. ${ }^{54,55}$ Healthy cells use the PD-1 pathway to prevent overstimulation of immune responses and to maintain immune tolerance of self-antigens. ${ }^{56-58}$ Tumor cells on the other hand often overexpress PD-L1 and PD-L2 on their surface, thus allowing the tumor cells to deactivate tumor-infiltrating activated T-cells. ${ }^{51,55}$ It has been postulated that the disruption of this interaction will lead to restored antitumor immunity. ${ }^{59-61}$ Nivolumab inhibits the interaction between PD-1 expressed on the Tcells and PD-L1/PD-L2 expressed on the tumor, thereby leading to T-cell mediated killing of the malignant cells. ${ }^{9}$

The CheckMate025 trial compared the effectiveness of the PD-1 inhibitor nivolumab versus everolimus in 821 patients with advanced clear-cell RCC who have previously been treated with up to two regimens of VEGF therapy. ${ }^{9}$ Patients were randomized to receive $3 \mathrm{mg}$ of nivolumab per kilogram of body weight intravenously every 2 weeks for $60 \mathrm{~min}$ or $10 \mathrm{mg}$ everolimus orally once a day. ${ }^{9}$ The primary endpoint of OS was 25.0 months and 19.6 months for nivolumab versus everolimus,

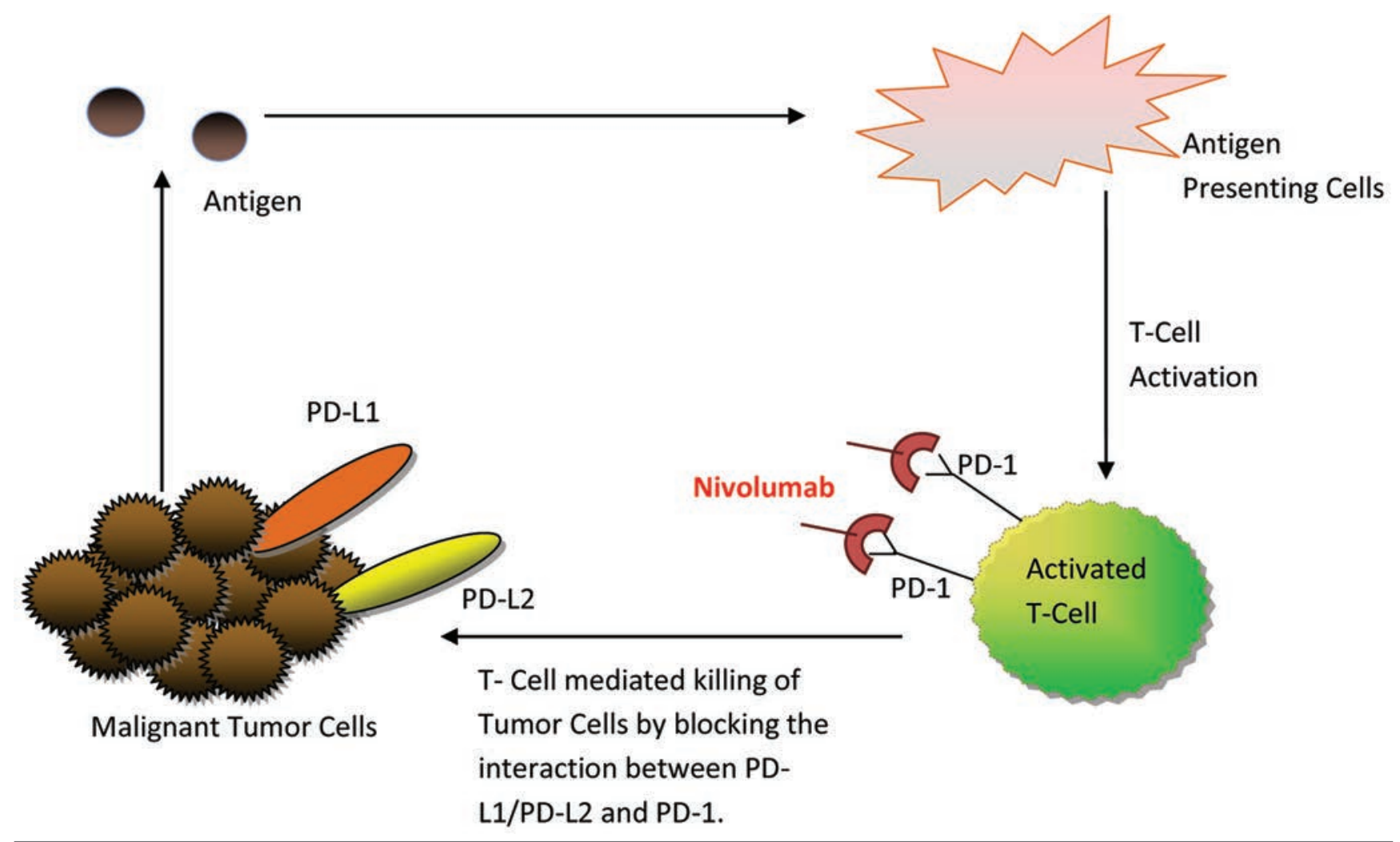

Figure 2. Mechanism of action of nivolumab: by blocking the interaction between programmed cell death-1 (PD-1) and PD-1 ligands (PD-L1/PD-L2), nivolumab assists with the induction of T-cell mediated killing of malignant cells. 
respectively. ${ }^{9}$ ORR was $25 \%$ for nivolumab and $5 \%$ for everolimus $(\mathrm{P}<0.001) .{ }^{9}$ Adverse events were less common in patients receiving nivolumab (19\%) than in patients on everolimus (37\%)..$^{9}$ The difference in PFS between the two agents was not statistically significant, possibly because patients treated with checkpoint inhibitors may exhibit pseudoprogression due to initial lymphocytic infiltration that may artificially make the tumors look slightly larger on imaging. ${ }^{9} 26 \%$ of patients receiving everolimus had at least 1 dose reduction, whereas dose reductions in the nivolumab group were not permitted. ${ }^{9}$ The most common adverse side-effects associated with nivolumab were nausea and pruritus. ${ }^{9}$

Furthermore, this trial examined the association between OS and tumor expression of PD-L1. ${ }^{9}$ Previous studies have identified PD-L1 expression to be associated with poor prognosis RCC and as a result PD-L1 expression has been postulated to improve OS post-nivolumab treatment. ${ }^{60-64}$ In the CheckMate 025 trial $92 \%$ of patients had quantifiable PD-L1 expression. ${ }^{9} 24 \%$ of patients with quantifiable PD-L1 expression had $\geq 1 \%$ PD-L1 expression, while $76 \%$ had $<1 \% .{ }^{9}$ A subgroup analysis displayed a median $\mathrm{OS}$ of 21.8 months $(\mathrm{N}=94)$ and 27.4 months $(\mathrm{N}=276)$ in patients with $\geq 1 \%$ PD-L1 expression and $<1 \%$ PDL1 expression, respectively. ${ }^{9}$ A median 0 S of 21.9 months $(\mathrm{N}=23)$ and 24.6 months $(\mathrm{N}=326)$ was observed in patients with $\geq 5 \%$ PD-L1 expression and $<5 \%$ PD-L1 expression, respectively. ${ }^{9}$ The results of this subgroup analysis suggest that PD-L1 expression is not a predictor of response to nivolumab treatment. ${ }^{65-67}$

Although results from the CheckMate025 trial are promising, the complete response rate or at least long term remission rate is still as low as with other targeted agents that are commonly used to treat mRCC. Additionally, the duration of treatment with nivolumab after pseudoprogression of mRCC tumors remains unclear and should be further examined. With the results of these recent trials, nivolumab, axitinib, and cabozantinib may become the standard second-line agents in the future, while moving everolimus beyond the third and fourth-line setting. Further studies will need to be conducted to directly compare the efficacy and safety of nivolumab versus cabozantinib as well as previously established agents such as axitinib. The possible relationship between PD-L1 expression as a predictive biomarker and survival outcome needs to be examined further, thereby optimizing treatment selection in the second-line setting for different subgroups of mRCC patients.

\section{Everolimus}

The mTOR inhibitor everolimus was the second-line standard of care until the introduction of cabozantinib and nivolumab, which demonstrated benefit over everolimus. In the pivotal RECORD-1 trial, RCC patients who previously progressed on sunitinib, sorafenib or both were randomized to daily oral everolimus at $10 \mathrm{mg}$ or placebo. ${ }^{68}$ The final results of the trial displayed a PFS of 4.9 months (everolimus) versus 1.9 months (placebo) $(\mathrm{P}<0.001)$ and an $0 \mathrm{~S}$ of 5.5 months (everolimus) versus 1.9 months (placebo) $(\mathrm{P}<0.001) .{ }^{69}$ As a result, everolimus has been used in second-line setting following prior TKI failure. ${ }^{69}$ The findings of both, the CheckMate025 and METEOR trials pushed Everolimus to the third-line setting and beyond. 8,9,68

A phase II trial examining the oral multi target fibroblast growth factor receptor and VEGFR TKI lenvatinib after prior treatment with VEGF-targeted therapy was compared against lenvatinib and everolimus combination therapy and against everolimus alone. ${ }^{70}$ The combination treatment of everolimus and lenvatinib recently received FDA approval in the United States for mRCC treatment. ${ }^{71}$ Results displayed a statistically significant increase in PFS in the combination group (14.6 months) and the stand-alone lenvatinib group (7.4 months) versus patients that received everolimus (5.5 months). ${ }^{70}$ There was no statistically significant difference in PFS between the lenvatinib and the combination group $(\mathrm{P}=0.12) .{ }^{70}$ Furthermore, this is the only combination that appears to significantly improve OS. The subsequent post hoc updated analysis displayed a significant difference in OS between patients in the combination group and those receiving everolimus only was seen [median OS 25.5 months $(95 \% \mathrm{CI}$ 16.4-NE) versus 15.4 months (95\% CI 11.8-19.6)]; (HR 0.51, 95\% CI $0.30-0.88, \mathrm{P}=0.024)$. The $\mathrm{OS}$ was not significantly different between patients on lenvatinib and everolimus or the combination group. Grade 3 or 4 events occurred in $50 \%$ of patients receiving everolimus, $79 \%$ of patients receiving single agent lenvatinib and $71 \%$ of the combination group. A phase III trial is warranted to confirm a substantial survival benefit in order to justify the financial costs and side effects of combination therapy. ${ }^{70}$ The combination therapy of lenvatinib and everolimus was granted accelerated approval due to FDA breakthrough therapy designation, which will require a post marketing confirmatory trial. ${ }^{71}$

\section{Sorafenib}

Sorafenib also remains a treatment option in later lines of therapy for advanced clear cell RCC. ${ }^{72}$ A randomized phase II trial of first-line sorafenib versus IFN- $\alpha$ showed no significantly different PFS between the two treatment groups. ${ }^{73}$ Sorafenib patients reported less toxicities and a better quality-of-life. ${ }^{72}$ Additionally, the randomized phase III INTORSECT trial examined sorafenib versus temsirolimus in second-line setting in mRCC patients who have previously progressed on sunitinib. ${ }^{74}$ Results displayed no significant PFS difference between the two treatment groups, however the sorafenib arm displayed a significantly larger $\mathrm{OS}$ of 16.6 months versus 12.3 months in the temsirolimus arm. ${ }^{74}$

\section{Third line therapy}

Currently, the GOLD and RECORD-1 trials are the only reported prospective randomized controlled trials evaluating third-line therapy in mRCC. ${ }^{68,75}$ In the GOLD trial dovitinib, an oral multi-target TKI inhibiting both VEGF and FGF was tested versus sorafenib. Dovitinib was given at $500 \mathrm{mg}$ orally 5 days on/2 days off, whereas sorafenib was given orally (400 mg twice daily). ${ }^{75}$ Results displayed no significant difference between median PFS of the two drugs. ${ }^{75}$ RECORD-1 tested the efficacy of everolimus $10 \mathrm{mg}$ daily versus placebo in patients with mRCC with a clear cell component. ${ }^{68}$ A subset analysis $(\mathrm{N}=108)$ of patients receiving third-line everolimus after previous treatment with VEGF-TKIs displayed a median PFS of 4.0 months in the everolimus group and 1.9 months in the placebo group. ${ }^{68}$ ORR were close to $0 \%$ for both groups and there was no significant difference in $0 S .68,69$ This indicates that there is activity of everolimus in the third line although the response rates are negligible, which is historically consistent with mTOR inhibitors as disease stabilizing agents.

A retrospective study further examined the use of third-line targeted therapy in mRCC patients $(\mathrm{N}=1012) .{ }^{14} 61.1 \%$ of patients displayed an ORR of stable disease or better. ${ }^{14}$ There was no statistically significant difference between OS or PFS between the different third-line agents, however a significant survival difference was observed when stratifying patients into the IMDC prognostic groups. ${ }^{14}$ An OS of 29.9, 15.5 and 5.5 months $(\mathrm{P}<0.0001)$ was observed in favorable, intermediate, and poor risk patients, respectively. ${ }^{14} \mathrm{~A}$ similar trend in PFS was observed, with favorable risk patients having the highest PFS of 7.5 months. ${ }^{14}$ Although there was no statistically significant difference associated between the different drug types, everolimus was most commonly used in $27.5 \%$ of the sample. ${ }^{14}$ With cabozantinib and nivolumab set to take the spot as primary agents in second-line therapy, everolimus will likely be more frequently used in the third-line setting or beyond. 


\section{Targeted therapy in non-clear cell renal cell carcinoma}

Although clear cell RCC is the most prevalent histology (75-80\%), the papillary type (10-15\%), chromophobe (5\%) and rare forms, such as collecting ducts carcinoma $(\leq 1 \%)$ also account for a significant portion of affected patients. ${ }^{76}$ As a result, the treatment for those patients has been understudied and treatments are generally adopted based on the results of trials examining the drug efficacies in clear cell RCC populations.

Currently, a variety of promising trials are testing different therapeutic combinations in non clear cell RCC. ${ }^{77}$ A phase II trial evaluating the efficacy of first-line AZD6094 (savolitinib, HMPL-504) in patients with papillary RCC is ongoing. ${ }^{78}$ Results from the ASPEN trial assessing everolimus versus sunitinib in patients with non-clear cell mRCC displayed prolonged radiographic PFS and higher rates of severe toxicities in the sunitinib group. ${ }^{79}$ Preliminary results from a prospective phase II trial display that pazopanib demonstrated a promising activity with a median PFS of 8.3 months in a non-clear cell cohort excluding collecting duct and sarcomatoid type RCC. ${ }^{80}$ Various clinical trials examining MET inhibitors, such as crizontinib and cabozantinib are also currently being tested particularly in papillary RCC. ${ }^{77}$ Other trials are examining the effects of cytotoxic chemotherapy, such as gemicitabine and doxorubicin in patients with RCC with sarcomatoid features. ${ }^{77}$ Lastly, the PD-L1 pathway is currently also being examined through the use of antibody atezolizumab (NCT01375842) in papillary and sarcomatoid histologies, thus displaying the potential for treatment with immunomodulatory agents in non-clear cell mRCC. ${ }^{81}$

\section{Optimization of targeted therapy sequencing in metastatic renal cell carcinoma}

With the sheer amount of therapeutic agents available for RCC, the sequence of the most optimal therapies must be examined (Figure 3). Since cross trial comparison is not a reliable method to examine differences between drugs, physicians tend to use safety profiles of the drugs when facilitating therapeutic selection. ${ }^{82}$ It remains critical to monitor and manage potential toxicities when treating $\mathrm{mRCC}$ patients with targeted agents. ${ }^{83}$ With the addition of nivolumab and cabozantinib the ideal sequence of targeted agents in the highly heterogeneous mRCC population needs to be elucidated. Physicians are often left to decide which drug to use in the mRCC patients until further trials establish a more definitive answer.

The CheckMate214 phase II clinical trial is currently examining the use of nivolumab and ipilumumab, a monoclonal antibody targeting CTLA-4 versus sunitinib in first-line setting. ${ }^{17}$ Depending on the results the nivolumab and ipilumumab combination may displace VEGFinhibitors as the standard of care first-line agent in the future. The mTOR, VEGF, MET/NEGF and PD-1 mechanisms could also be targeted using a combination of the currently available targeted therapies. Results from the CheckMate016 (NCT01472081) phase I trial examining nivolumab together with VEGF inhibitors sunitinib or pazopanib showed encouraging anti-tumor activity as well as a manageable safety profile of combination therapy in mRCC patients. ${ }^{84}$

The use of biomarkers in $\mathrm{mRCC}$ will be critical in the future. Future treatment options may be directed by the presence of positive biomark-

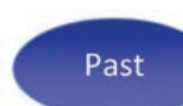

ast

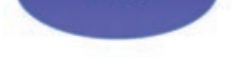

$\sum$
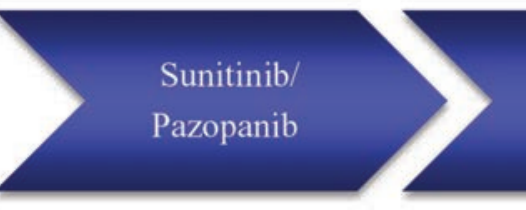

Everolimus/

Axitinib
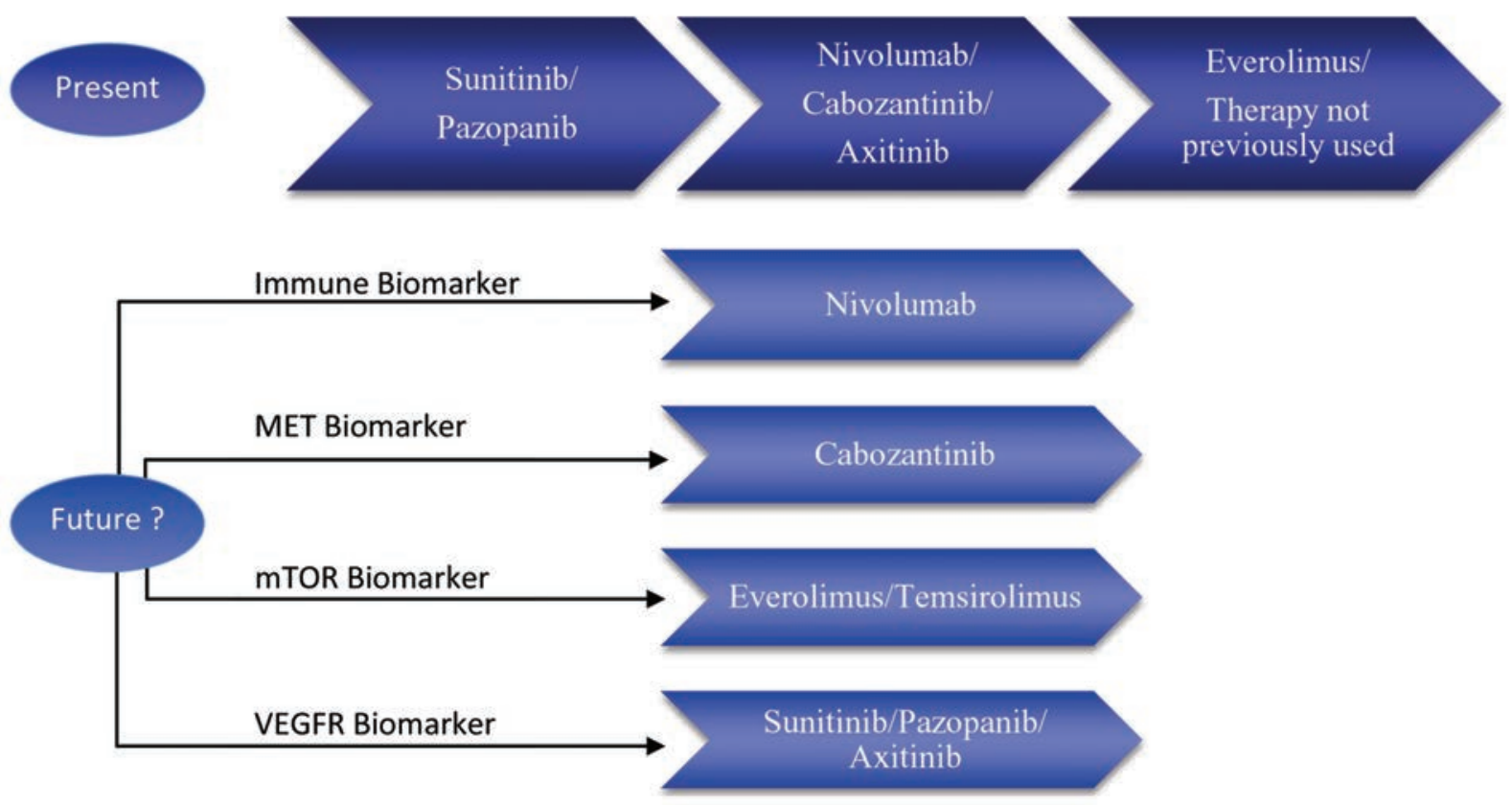

Figure 3. Past, current and future possibilities for renal cell carcinoma treatments. mTOR, mammalian target of rapamycin; VEGFR, vascular endothelial growth factor receptor. 
ers, thereby optimizing treatment selection for patients. A hypothetical future treatment paradigm may have patients with up-regulated MET receive cabozantinib, a positive immune marker would prompt the use of nivolumab or other immunomodulatory agents, patients with upregulated MTOR would receive everolimus or temsirolimus, while patients with VEGFR upregulation may be more susceptible to sunitinib, pazopanib or axitinib treatment. These biomarkers may be discovered and used in the future and will require further validation. The identification of novel biomarkers and the usage of these biomarkers in the clinical setting will be critical to personalizing targeted therapies in mRCC. It is important to note that therapeutic choices are heavily influenced by funding availability and approved guidelines, thus depending on the institution patients may receive different interventions.

\section{Conclusions}

The treatment of mRCC continues to evolve faster than ever before. New targeted agents have been developed with treatment regimens continuing to be optimized. Novel agents, such as cabozantinib attempt to further prevent angiogenesis while other novel drugs such as nivolumab inhibit the immune checkpoint PD-1 pathway. A large number of clinical trials are ongoing to help elucidate the best therapy for patients with non-clear cell RCC.

\section{References}

1. Canadian Cancer Society's Advisory Committee on Cancer Statistics. Canadian Cancer Statistics 2015. Toronto, ON: Canadian Cancer Society; 2015. Available from: https:/www.cancer.ca/ / media/cancer.ca/CW/cancer\%20information/cancer\%20101/Canadi an\%20cancer\%20statistics/Canadian-Cancer-Statistics-2015EN.pdf

2. Ljungberg B, Campbell SC, Choi HY, et al. The epidemiology of renal cell carcinoma. Eur Urol 2011;60:615-21.

3. North S, Naveen B, Basiuk J, et al. Management of advanced kidney cancer: Canadian Kidney Cancer Forum consensus update. CUAJ 2015;9:164-70.

4. Motzer RJ, Bander NH, Nanus DM. Renal-cell carcinoma. N Engl J Med 1996;335:865-75.

5. Czarnecka AM, Kornakiewicz A, Kukwa W, et al. Frontiers in clinical and molecular diagnostics and staging of metastatic clear cell renal cell carcinoma. Future Oncol 2014;10:1095-111.

6. Battelli C, Cho DC. mTOR inhibitors in renal cell carcinoma. Therapy 2011;8:359-67.

7. Choueiri TK. VEGF inhibitors in metastatic renal cell carcinoma: current therapies and future perspective. Curr Clin Pharmacol 2011;6:164-8.

8. Choueiri TK, Escudier B, Powles T, et al. Cabozantinib versus everolimus in advanced renal-cell carcinoma. N Engl J Med 2015; 373:1814-23.

9. Motzer RJ, Escudier B, McDermott DF, et al. Nivolumab versus everolimus in advanced renal-cell carcinoma. N Engl J Med 2015; 373:150925150201006.

10. Heng DYC, Xie W, Regan MM, et al. Prognostic factors for overall survival in patients with metastatic renal cell carcinoma treated with vascular endothelial growth factor-targeted agents: results from a large, multicenter study. J Clin Oncol 2009;27:5794-9.

11. Li H, Samawi H, Heng DYC. The use of prognostic factors in metastatic renal cell carcinoma. Urol Oncol 2015;33:509-16.

12. Motzer RJ, Mazumdar M, Bacik J, et al. Survival and prognostic stratification of 670 patients with advanced renal cell carcinoma. J Clin Oncol 1999;17:2530-40.

13. Ko JJ, Xie W, Kroeger N, et al. The International Metastatic Renal Cell Carcinoma Database Consortium model as a prognostic tool in patients with metastatic renal cell carcinoma previously treated with first-line targeted therapy: a population-based study. Lancet Oncol 2015;16:293-300.

14. [No authors listed]. Fourteenth International Kidney Cancer Symposium Miami Marriott Biscayne Bay, Miami, Florida 6-7 November 2015. BJU Int 2015;116:1-23.

15. Heng DYC, Xie W, Regan MM, et al. External validation and comparison with other models of the International Metastatic RenalCell Carcinoma Database Consortium prognostic model: a population-based study. Lancet Oncol 2013;14:141-8.

16. ClinicalTrials.gov. Phase 3 trial of autologous dendritic cell immunotherapy (AGS-003) plus standard treatment of advanced renal cell carcinoma (RCC). ClinicalTrials.gov Identifier: NCT01582672. Available from: https://clinicaltrials.gov/ct2/show/ NCT01582672

17. ClinicalTrials.gov. Nivolumab combined with ipilimumab versus sunitinib in previously untreated advanced or metastatic renal cell carcinoma (CheckMate 214). ClinicalTrials.gov Identifier: NCT02231749. Available from: https:/clinicaltrials.gov/ct2/show/ NCT02231749

18. Hudes G, Carducci M, Tomczak P, et al. Temsirolimus, interferon alfa, or both for advanced renal-cell carcinoma. N Engl J Med 2007; 356:2271-81.

19. Fisher R, Pender A, Thillai K, et al. Observation as a treatment strategy for advanced renal cell carcinoma-a call for prospective validation. Front Oncol 2012;2:155.

20. Motzer RJ, Hutson TE, Tomczak P, et al. Sunitinib versus interferon alfa in metastatic renal-cell carcinoma. N Engl J Med 2007;356: 115-24.

21. Rini BI, Dorff TB, Elson P, et al. A prospective observational study of metastatic renal cell carcinoma (mRCC) prior to initiation of systemic therapy. J Clin Oncol 2014;32:5s, (suppl; abstr 4520).

22. Park I, Lee J-L, Ahn J-H, et al.Active surveillance for metastatic or recurrent renal cell carcinoma. J Cancer Res Clin Oncol 2014;140: 1421-8.

23. Matsubara N, Mukai H, Naito Y, et al. First experience of active surveillance before systemic target therapy in patients with metastatic renal cell carcinoma. Urology 2013;82:118-23.

24. Heng DYC, Wells JC, Rini BI, et al. Cytoreductive nephrectomy in patients with synchronous metastases from renal cell carcinoma: results from the International Metastatic Renal Cell Carcinoma Database Consortium. Eur Urol 2014;66:704-10.

25. Mendel $\mathrm{DB}$, Laird $\mathrm{AD}$, Xin $\mathrm{X}$, et al. In vivo antitumor activity of SU11248, a novel tyrosine kinase inhibitor targeting vascular endothelial growth factor and platelet-derived growth factor receptors: determination of a pharmacokinetic/pharmacodynamic relationship. Clin Cancer Res 2003;9:327-37.

26. Bjarnason GA, Knox JJ, Kollmannsberger CK, et al. Phase II study of individualized sunitinib as first-line therapy for metastatic renal cell cancer (mRCC). J Clin Oncol 2015;33, (suppl; abstr 4555).

27. Sternberg CN, Davis ID, Mardiak J, et al. Pazopanib in locally advanced or metastatic renal cell carcinoma: results of a randomized phase III trial. J Clin Oncol 2010;28:1061-8.

28. Motzer RJ, Hutson TE, Cella D, et al. Pazopanib versus sunitinib in metastatic renal-cell carcinoma. N Engl J Med 2013;369:722-31.

29. Escudier B, Porta C, Bono P, et al. Randomized, controlled, doubleblind, cross-over trial assessing treatment preference for pazopanib versus sunitinib in patients with metastatic renal cell carcinoma: PISCES Study. J Clin Oncol 2014;32:1412-8.

30. Rini BI, Halabi S, Rosenberg JE, et al. Bevacizumab plus interferon 
alfa compared with interferon alfa monotherapy in patients with metastatic renal cell carcinoma: CALGB 90206. J Clin Oncol 2008; 26:5422-8.

31. Rini BI, Escudier B, Tomczak P, et al. Comparative effectiveness of axitinib versus sorafenib in advanced renal cell carcinoma (AXIS): a randomised phase 3 trial. Lancet (London, England) 2011;378: 1931-9.

32. Rini B, Rixe 0, Bukowski R, et al. AG-013736, a multi-target tyrosine kinase receptor inhibitor, demonstrates anti-tumor activity in a Phase 2 study of cytokine-refractory, metastatic renal cell cancer (RCC). ASCO Meet Abstr 2005;23:4509.

33. Sonpavde G, Hutson TE, Rini BI. Axitinib for renal cell carcinoma. Expert Opin Investig Drugs 2008;17:741-8.

34. Motzer RJ, Escudier B, Tomczak P, et al. Axitinib versus sorafenib as second-line treatment for advanced renal cell carcinoma: overall survival analysis and updated results from a randomised phase 3 trial. Lancet Oncol 2013;14:552-62.

35. Wu X, Liu X, Koul S, et al. AXL kinase as a novel target for cancer therapy. Oncotarget 2014;5:9546-63.

36. Currie MJ, Gunningham SP, Turner K, et al. Expression of the angiopoietins and their receptor Tie2 in human renal clear cell carcinomas; regulation by the von Hippel-Lindau gene and hypoxia. $\mathrm{J}$ Pathol 2002;198:502-10.

37. Grüllich C. Cabozantinib: a MET, RET, and VEGFR2 tyrosine kinase inhibitor. Recent Results Cancer Res 2014;201:207-14.

38. Yakes FM, Chen J, Tan J, et al. Cabozantinib (XL184), a novel MET and VEGFR2 inhibitor, simultaneously suppresses metastasis, angiogenesis, and tumor growth. Mol Cancer Ther 2011;10:2298-308.

39. Zhou L, Liu X-D, Sun M, et al. Targeting MET and AXL overcomes resistance to sunitinib therapy in renal cell carcinoma. Oncogene 2016;35:2687-97.

40. Sennino B, Ishiguro-Oonuma T, Wei Y, et al. Suppression of tumor invasion and metastasis by concurrent inhibition of c-Met and VEGF signaling in pancreatic neuroendocrine tumors. Cancer Discov 2012;2:270-87.

41. Baldewijns MM, van Vlodrop IJH, Vermeulen PB, et al. VHL and HIF signalling in renal cell carcinogenesis. J Pathol 2010;221:125-38.

42. Banumathy G, Cairns P. Signaling pathways in renal cell carcinoma. Cancer Biol Ther 2014;10:658-64.

43. Sierra JR, Tsao M-S. c-MET as a potential therapeutic target and biomarker in cancer. Ther Adv Med Oncol 2011;3:S21-35.

44. Rini BI, Flaherty K. Clinical effect and future considerations for molecularly-targeted therapy in renal cell carcinoma. Urol Oncol 2008;26:543-9.

45. Rini BI, Atkins MB. Resistance to targeted therapy in renal-cell carcinoma. Lancet Oncol 2009;10:992-1000.

46. Loges S, Schmidt T, Carmeliet P. Mechanisms of resistance to antiangiogenic therapy and development of third-generation antiangiogenic drug candidates. Genes Cancer 2010;1:12-25.

47. Shojaei F, Lee JH, Simmons BH, et al. HGF/c-Met acts as an alternative angiogenic pathway in sunitinib-resistant tumors. Cancer Res 2010;70:10090-100.

48. Gibney GT, Aziz SA, Camp RL, et al. c-Met is a prognostic marker and potential therapeutic target in clear cell renal cell carcinoma. Ann Oncol 2013;24:343-9.

49. Elisei R, Schlumberger MJ, Müller SP, et al. Cabozantinib in progressive medullary thyroid cancer. J Clin Oncol 2013;31:3639-46.

50. Choueiri TK, Powles T, Escudier BJ, et al. Overall survival (OS) in METEOR, a randomized phase 3 trial of cabozantinib (Cabo) versus everolimus (Eve) in patients (pts) with advanced renal cell carcinoma (RCC). J Clin Oncol 2016;34, (suppl; abstr 4506).

51. Hamanishi J, Mandai M, Iwasaki M, et al. Programmed cell death 1 ligand 1 and tumor-infiltrating CD8+ T lymphocytes are prognostic factors of human ovarian cancer. Proc Natl Acad Sci U S A 2007; 104:3360-5.
52. Hamid 0, Carvajal RD. Anti-programmed death-1 and anti-programmed death-ligand 1 antibodies in cancer therapy. Expert Opin Biol Ther 2013;13:847-61.

53. Chen L, Flies DB. Molecular mechanisms of T cell co-stimulation and co-inhibition. Nat Rev Immunol 2013;13:227-42.

54. Homet Moreno B, Ribas A. Anti-programmed cell death protein-1/ligand-1 therapy in different cancers. Br J Cancer 2015;112:1421-7.

55. McDermott DF, Atkins MB. PD-1 as a potential target in cancer therapy. Cancer Med 2013;2:662-73.

56. Freeman GJ, Long AJ, Iwai Y, et al. Engagement of the PD-1 immunoinhibitory receptor by a novel B7 family member leads to negative regulation of lymphocyte activation. J Exp Med 2000;192: 1027-34.

57. Keir ME, Liang SC, Guleria I, et al. Tissue expression of PD-L1 mediates peripheral T cell tolerance. J Exp Med 2006;203:883-95.

58. Korman AJ, Peggs KS, Allison JP. Checkpoint blockade in cancer immunotherapy. Adv Immunol 2006;90:297-339.

59. Motzer RJ, Rini BI, McDermott DF, et al. Nivolumab for metastatic renal cell carcinoma: results of a randomized phase II trial. J Clin Oncol 2015;33:1430-7.

60. Topalian SL, Hodi FS, Brahmer JR, et al. Safety, activity, and immune correlates of anti-PD-1 antibody in cancer. N Engl J Med 2012;366:2443-54.

61. Brahmer JR, Drake CG, Wollner I, et al. Phase I study of singleagent anti-programmed death-1 (MDX-1106) in refractory solid tumors: safety, clinical activity, pharmacodynamics, and immunologic correlates. J Clin Oncol 2010;28:3167-75.

62. Thompson RH, Gillett MD, Cheville JC, et al. Costimulatory B7-H1 in renal cell carcinoma patients: Indicator of tumor aggressiveness and potential therapeutic target. Proc Natl Acad Sci U S A 2004;101: 17174-9.

63. Thompson RH, Kuntz SM, Leibovich BC, et al- Tumor B7-H1 is associated with poor prognosis in renal cell carcinoma patients with long-term follow-up. Cancer Res 2006;66:3381-5.

64. Choueiri TK, Fishman MN, Escudier B, et al. Immunomodulatory activity of nivolumab in metastatic renal cell carcinoma (mRCC): Association of biomarkers with clinical outcomes. ASCO Meet Abstr 2015;33:4500.

65. Brahmer J, Reckamp KL, Baas P, et al. Nivolumab versus docetaxel in advanced squamous-cell non-small-cell lung cancer. $\mathrm{N}$ Engl $\mathrm{J}$ Med 2015;373:123-35.

66. Borghaei H, Paz-Ares L, Horn L, et al. Nivolumab versus docetaxel in advanced nonsquamous non-small-cell lung cancer. $\mathrm{N}$ Engl $\mathrm{J}$ Med 2015;373:1627-39.

67. Robert C, Long G V., Brady B, et al. Nivolumab in previously untreated melanoma without BRAF mutation. N Engl J Med 2014; 372:141116004513004.

68. Motzer RJ, Escudier B, Oudard S, et al. Efficacy of everolimus in advanced renal cell carcinoma: a double-blind, randomised, placebo-controlled phase III trial. Lancet (London, England) 2008;372:449-56.

69. Motzer RJ, Escudier B, Oudard S, et al. Phase 3 trial of everolimus for metastatic renal cell carcinoma. Cancer 2010;116:4256-65.

70. Motzer RJ, Hutson TE, Glen H, et al. Lenvatinib, everolimus, and the combination in patients with metastatic renal cell carcinoma: a randomised, phase 2, open-label, multicentre trial. Lancet Oncol 2015;16:1473-82.

71. U.S. Food and Drug Administration (FDA). Lenvatinib in combination with everolimus; last updated: May 16, 2016. Available from: http://www.fda.gov/Drugs/InformationOnDrugs/ApprovedDrugs/uc m501070.htm

72. Escudier B, Eisen T, Stadler WM, et al. Sorafenib in advanced clearcell renal-cell carcinoma. N Engl J Med 2007;356:125-34.

73. Escudier B, Szczylik C, Hutson TE, et al. Randomized phase II trial 
of first-line treatment with sorafenib versus interferon alfa-2a in patients with metastatic renal cell carcinoma. J Clin Oncol 2009;27:1280-9.

74. Hutson TE, Escudier B, Esteban E, et al. Randomized phase III trial of temsirolimus versus sorafenib as second-line therapy after sunitinib in patients with metastatic renal cell carcinoma. J Clin Oncol 2014;32:760-7.

75. Motzer RJ, Porta C, Vogelzang NJ, et al. Dovitinib versus sorafenib for third-line targeted treatment of patients with metastatic renal cell carcinoma: an open-label, randomised phase 3 trial. Lancet Oncol 2014;15:286-96.

76. Cairns P. Renal cell carcinoma. Cancer Biomark 2010;9:461-73.

77. Valenca LB, Hirsch MS, Choueiri TK, et al. Non-clear cell renal cell carcinoma, part 2: therapy. Clin Adv Hematol Oncol 2015;13:383-91.

78. ClinicalTrials.gov. A phase II trial to evaluate the efficacy of AZD6094 (HMPL-504) in patients with papillary renal cell carcinoma (PRCC). ClinicalTrials.gov Identifier: NCT02127710. Available from: https://clinicaltrials.gov/ct2/show/NCT02127710

79. Armstrong AJ, Broderick S, Eisen T, et al. Final clinical results of a randomized phase II international trial of everolimus vs. sunitinib in patients with metastatic non-clear cell renal cell carcinoma (ASPEN). J Clin Oncol 2015;33, (suppl; abstr 4507).

80. Jung KS, Cho J, Yoo KH, et al. Pazopanib for treatment of metastatic renal cell carcinoma with non-clear cell histology: single-arm, open label, multicenter, phase II study. J Clin Oncol 2016;34, (suppl $2 \mathrm{~S}$; abstr 577).

81. ClinicalTrials.gov. A phase 1 study of MPDL3280A (an engineered anti-PDL1 antibody) in patients with locally advanced or metastatic solid tumors. ClinicalTrials.gov Identifier: NCT01375842Available from: https://clinicaltrials.gov/ct2/show/NCT01375842

82. Pal SK, Choueiri TK, Karam JA, et al. Metastatic renal cell carcinoma: contending with a sea change in therapy. Urol Oncol 2015;33:507-8.

83. Pham A, Ye D-W, Pal S. Overview and management of toxicities associated with systemic therapies for advanced renal cell carcinoma. Urol Oncol 2015;33:517-27.

84. Amin A, Plimack ER, Infante JR, et al. Nivolumab (anti-PD-1; BMS936558, ONO-4538) in combination with sunitinib or pazopanib in patients (pts) with metastatic renal cell carcinoma (mRCC). J Clin Oncol 2014;32:5s, (suppl; abstr 5010). 